\title{
PROGRAMA SAÚDE DA FAMÍLIA: DESAFIOS E POTENCIALIDADES FRENTE AO USO DE DROGAS
}

\author{
FAMILY HEALTH PROGRAM: CHALLENGES AND POTENTIALITIES REGARDING DRUGS USE
} PROGRAMA SALUD DE LA FAMILIA: DESAFIOS Y POTENCIALIDADES ANTE EL USO DE DROGAS

\section{Marcelle Aparecida de Barros ${ }^{1}$, Sandra Cristina Pillon ${ }^{2}$}

RESUMO: Os estudos epidemiológicos sobre o consumo de drogas apontam que este fenômeno tem ganhado um problema dimensional na saúde pública. Atualmente, o Programa Saúde da Família (PSF) é apresentado pelo Ministério da Saúde como modelo de reestruturação da assistência à saúde na atenção primária, o qual tem como objetivo oferecer uma assistência centrada na família, permeadas pela integralidade, resolutividade e vínculos comunitários. O objetivo deste artigo é discutir as possibilidades de atuação dos profissionais de saúde do Programa Saúde da Família frente aos pacientes usuários de drogas. É evidente que, ao contrário de outras ações já desenvolvidas pelos profissionais do PSF em outras áreas da atenção à saúde, vem se mostrando de maneira clara e objetiva. Tal fato é observado nas atividades cotidianas do profissional no PSF, que pouco se aborda quanto aos problemas relacionados ao uso de drogas. No entanto, os estudos enfatizam que existe uma gama de possibilidades para a atuação desses profissionais no PSF. Embora outros estudos evidenciem as fragilidades dessa equipe frente à assistência aos usuários de drogas, porém que estas podem ser superadas mediante um investimento na capacitação e valorização da equipe.

PALAVRAS CHAVES: Programa Saúde da Família; Drogas Ilícitas; Conhecimentos, Atitudes e Práticas em Saúde.

ABSTRACT: Epidemiological studies on drugs use point towards this phenomenon as a public health problem. Nowadays, the Family Health Program (FHP) is presented by the Health Ministry as a model to restructure primary health care and aims to offer family-centered care, permeated by integrality, problem solving and community bonds. This article aims to discuss action possibilities of Family Health Care professionals involving drugs patients. It is evident that, as opposed to other actions already developed by FHP professionals in other health care areas, which has appeared clearly and objectively. This fact is observed in the daily activities of FHP professionals, which give little attention to drugsrelated problems. However, research emphasizes that there exists a broad range of action possibilities for FHP professionals. Although other studies evidence this team's fragilities in terms of care for drugs users, these can be overcome by investing in the training and valuation of these professionals.
KEY WORDS: Family Health Program; Street drugs; Health Knowledge, Attitudes, Practice.

RESUMEN: Los estudios epidemiológicos sobre el consumo de drogas indican que este fenómeno se volvió un problema dimensional en la salud pública. Hoy día, el Programa Salud de la Familia (PSF) es presentado por el Ministerio de la Salud como modelo para la reestructuración de la atención a la salud en la atención primaria. Tiene como objetivo ofrecer una atención centrada en la familia y es permeada por la integralidad, resolutividad y vínculos comunitarios. La finalidad de este artículo es discutir las posibilidades de actuación de los profesionales de salud del PSF ante los pacientes usuarios de drogas. Es evidente que, al contrario de otras acciones desarrolladas por los profesionales del PSF en otras áreas de atención a la salud, viene mostrándose de manera clara y objetiva. Tal hecho se observa en las actividades cotidianas del profesional en el PSF, que poco se trata de los problemas relacionados al uso de drogas. Sin embargo, los estudios destacan que existe una gama de posibilidades para la actuación de esos profesionales en el PSF. Aunque otros estudios evidencien las fragilidades de eso equipo ante la atención a los usuarios de drogas, estas pueden ser superadas mediante una inversión en la capacitación y valorización del equipo.

PALABRAS CLAVES: Programa Salud de la Familia; Drogas ilícitas; Conocimientos, Actitudes y Práctica en Salud.

1 Enfermeira. Mestre em Enfermagem Psiquiátrica. Docente da Disciplina de Saúde Coletiva. Enfermagem do UniSalesiano - Centro Universitário Católico Auxilium Araçatuba -SP.End. Rua Nova Luzitânia, 168, Bairro: N.S. Aparecida, Araçatuba-SP, cep: 16.056-630. E-mail: marcellebarros@ibest.com.br

2 Professora Doutora. Departamento de Enfermagem Psiquiátrica e Ciências Humanas Escola de Enfermagem de Ribeirão Preto. Universidade de São Paulo EERP.USP. Centro Colaborador da OMS, para o Desenvolvimento da Pesquisa em Enfermagem.End: Avenida Bandeirantes, 3.900, Campus Universitário USP, Monte Alegre, cep: 14.040-902, Ribeirão Preto -SP. Email: pillon@eerp.usp.br. 


\section{INTRODUÇÃO}

A Organização Mundial da Saúde (2002) identificou que o uso do cigarro, do álcool e das drogas ilícitas está entre os 20 maiores problemas de saúde no mundo. Ainda, este órgão estima que o tabaco é responsável por $9 \%$ dos casos de morte, o que corresponde a $4,1 \%$ da carga global de todas as doenças. Esta estimativa é medida baseando-se no número de anos gasto com a doença (Disability Adjusted Life Years - DALYS), enquanto o álcool é responsável por $3.2 \%$ das mortes e $4.0 \%$ do DALYs (OMS, 2002).

Estes índices são preocupantes, pois sabemos que o abuso dessas substâncias, geralmente vem acompanhado pelos problemas sociais e de saúde, econômicos, jurídicos e legais, que também envolvem as diversas conseqüências nas relações pessoais do indivíduo no meio em que está envolvido.

Além disso, os pacientes fumantes, ou usuários de álcool e/ou de drogas têm usado estes serviços com mais freqüência. Ressalta nesse manual, que os profissionais da atenção primária têm oportunidades únicas de intervir nas fases prévias ao desenvolvimento de problemas do uso e mesmo da dependência (HENRY-EDWARDS et al, 2003).

Quanto ao enfrentamento dos problemas nos âmbitos da saúde, a Organização Mundial da Saúde formulou um manual para profissionais da atenção primária que visa a intervenção breve para o uso de substâncias referência que nos países desenvolvidos. Pelo menos uma vez ao ano, $8 \%$ da população tem procurado assistência de saúde na atenção primária.

Neste sentido, a literatura internacional (AALTO, 2002) segue a proposta da OMS no que tange a assistência na atenção primária, que esta esteja voltada para a promoção de saúde e prevenção de problemas relacionados ao uso de substâncias psicoativas, neste caso. O consenso destes estudos é que se promova a identificação precoce dos referidos problemas e aplicação das intervenções breves, como oportunidade máxima do profissional na oferta de uma assistência a estes pacientes.

Diante estes aspectos, apresentamos neste texto uma revisão dos desafios que o Brasil vem enfrentando por meio do Programa Saúde da Família, apresentando suas facilidades e dificuldades no enfrentamento dos problemas relacionados ao uso de drogas na atenção primária, por meio do Programa de Saúde da Família. Tal fato evoluiu muito nos últimos anos e muito ainda tem que caminhar nessa área tão complexa e desafiadora que é o uso das substâncias psicoativas.

\footnotetext{
O Programa Saúde da Família na atenção primária à saúde.
}

No Brasil, a atenção primária à saúde que atualmente o Programa Saúde da Família está inserido, é considerado a "porta de entrada" do Sistema Único de Saúde. Local que deveria e está assumindo também, a posição de assistência na atenção aos usuários de álcool e de outras drogas.

De acordo com VIANA \& DAL POZ (1998), pode-se considerar que o PSF iniciou a partir do Programa dos Agentes Comunitários da Saúde (PACS) instituído pelo Ministério da Saúde, com uma proposta centrada na assistência à família como unidade de ação programática de saúde, deslocando a atenção do indivíduo para a família. O PACS também introduziu uma visão ativa de ação preventiva em saúde, que busca estabelecer uma verdadeira interação com a comunidade e, ao mesmo tempo, reorganizando, a demanda por assistência.

O programa apresenta-se como uma possibilidade de reestruturação da atenção primária, como destacada por TRAD \& BASTOS (1998), a partir de um conjunto de ações conjugadas em sintonia com os princípios de territorialização, intersetorialidade, descentralização, coresponsabilização e priorização de grupos populacionais com maior risco de adoecer ou morrer.

Referente a dinâmica de funcionamento cada equipe do PSF atende de 600 a 1.000 famílias ou 4.500 pessoas numa área de abrangência restrita e limitada. São compostas por, um médico generalista, ou médico da família, um enfermeiro, um auxiliar de enfermagem e cinco ou seis agentes comunitários de saúde.

De acordo com LEYCOVITZ \& GARRIDO (1996), o PSF é um modelo de atenção que reforça a idéia de saúde como um direito de cidadania, e que este modelo deve oferecer serviços mais resolutivos, integrais e humanizados, pautados na intersetorialidade e da participação da comunidade nas ações de saúde e no controle social.

SOUZA (1999, p.24) destaca alguns compromissos importantes da prática da Saúde da Família como espaço social familiar como núcleo básico da abordagem, assistência integral, resolutiva, contínua e de qualidade, intervenção em fatores de risco, humanização da assistência, criação de vínculos e co-responsabilidade entre profissionais e a comunidade, desenvolvimento de ações intersetoriais através de parcerias; democratização do processo saúde-doença, da organização do serviço, da produção social da saúde como direito da cidadania e organização da comunidade para efetivo exercício do controle social.

Muitos são os esforços e avanços do Programa Saúde da Família na assistência à saúde da população, como indicou a pesquisa "Avaliação do Processo de Implantação e de Funcionamento do 
PSF", Brasil em 1999 (2003a). A exemplo disso está o tratamento de pacientes hipertensos que atingiu índices surpreendentes passando de 34,8\% para $98,5 \%$ e no acompanhamento do crescimento e desenvolvimento infantil (53,1\% para $98,3 \%)$ no Brasil onde o programa foi implantado.

Porém, ao contrário de outras ações desenvolvidas pelos profissionais do PSF em outras áreas de atenção à saúde, que já há tempo vem se mostrando de maneira clara e objetiva, o que se observa cotidianamente é que muito pouco se aborda quanto ao abuso e dependência das drogas na prática do profissional no PSF.

No momento em que o Ministério da Saúde, BRASIL (2001), considera o Programa Saúde da Família como um modelo de reorganização da prática assistencial criada para substituir o modelo tradicional centrado no hospital, ele assume o desafio de garantir o acesso igualitário a todos os serviços de saúde. Mediante essa relevância, o PSF fechou o ano de 2002 com 16.698 equipes, BRASIL, (2003b) equivalentes a 55 milhões de brasileiros assistidos em 4.161 municípios (aproximadamente 32\% da população).

Considerando que, a meta do Ministério da Saúde é multiplicar o número de equipes do programa nos próximos quatro anos, pode-se imaginar o impacto das ações desenvolvidas pelos profissionais do PSF nas condições de saúde da população brasileira, e entendendo que entre as ações que serão desenvolvidas pelo PSF na atenção básica a saúde, deve estar incluída as ações de assistência ao abuso e a dependência de drogas.

Assim, é inequívoca a necessidade de trazer à luz esta questão enquanto desafio profissional do PSF.

\section{O Programa Saúde da Família: possibilidades de enfrentamento na problemática das drogas.}

A importância da articulação entre a atenção primária, a saúde mental e a atenção aos usuários de álcool e outras drogas vem, ultimamente, sendo enfatizada com o propósito de modificações da prática assistencial visando a sua inserção e a integração dessa tríade por parte da Organização Mundial de Saúde e do Ministério da Saúde.

De fato, essa vinculação se torna imprescindível nos dias atuais, porém, o Ministério da Saúde, BRASIL (2003c), considera que, embora sejam sempre importante e necessária às articulações da saúde mental com a atenção básica, nem sempre a esta apresenta condições favoráveis para realizar essa difícil tarefa; às vezes, pela falta de recursos, e ou pela falta de capacitação que acabam por prejudicar o desenvolvimento de uma ação integral pelas equipes.
Em corroboração com este contexto, um estudo de GONÇALVES (2002) sobre a prática de cuidados relacionados ao abuso e dependência de drogas no cotidiano de uma equipe do Programa Saúde da Família encontrou graves problemas relacionados ao uso de substâncias que se estendem além das questões individuais, aos familiares e a comunidade. Essa autora desenvolveu uma ação reflexão junto a essa equipe a fim de superar esses problemas no cotidiano de trabalho.

Os resultados dessa pesquisa evidenciaram as fragilidades e potencialidades técnicas do grupo no encaminhamento das dificuldades superadas, mediante um investimento na capacitação da equipe e na criação de um projeto de ação comunitária, compartilhado com lideranças da comunidade.

GONÇALVES (2002) aborda que o abuso de drogas não pode ser examinado de forma isolada, pois está em centrado contexto complexo e dinâmico. Os profissionais do PSF, por terem a família como unidade programática de ações e cuidados, devem procurar a construção de atitudes de solidariedade e compreensão, para ajudar a prevenir e tratar as conseqüências que o consumo de substâncias acarreta na família e na comunidade.

Neste estudo, a autora enfatiza que a prática de cuidados relacionados ao abuso e dependência de drogas no PSF deve priorizar medidas seletivas de prevenção e de prevenção primária por meio de ações de promoção da saúde, envolvendo o indivíduo, as famílias, os grupos e a comunidade na criação de condições de redução da demanda de oferta de drogas. Sendo que, o que deve fazer a diferença na atuação do Programa Saúde da Família é a criatividade, a inovação, a qualidade, a integralidade, a singularidade da assistência oferecida pelos profissionais da equipe de saúde.

Esse estudo vem reiterar que, embora os profissionais da atenção primária não estejam preparados em suas habilidades teórico-práticas para manejar com essa questão; uma vez se investindo, valorizando, capacitando e explorando as potencialidades dos profissionais da atenção primária à saúde, estes podem contribuir efetivamente no âmbito da assistência aos usuários de drogas.

Dessa maneira, o estabelecimento de ações intersetoriais deve permitir que, em cada área, surjam contribuições para a solução dos problemas de saúde que emergem de discussões comunitárias. As prioridades poderão assim transformar-se em pautas positivas, promotoras de saúde. E a educação e a transformação para a saúde passam a ser fundamental nesse processo na medida em que aumentam a conscientização dos comportamentos de saúde dos cidadãos e intensificam a participação dos mesmos na definição das prioridades (CAMPOS, 2003). Ainda, este autor, reforça que a equipe não 
tem necessariamente de dominar todo o conhecimento sobre as múltiplas ocorrências e agravos que acometem na comunidade, mas sim estar atenta às possíveis relações existentes entre o adoecer e a vida cotidiana de seus integrantes.

Existe uma ampla e variada gama de possibilidades em que os profissionais da atenção primária podem atuar frente a esta temática. Atuando como, por exemplo, nos três níveis de prevenção.

Porém, é sempre importante destacar que ao se dar prioridade à prevenção, não se pode descuidar do atendimento das demais necessidades de saúde, nos níveis mais complexos do sistema, ao se ter em mente os objetivos e pressupostos do Sistema Único de Saúde, que devem assegurar o acesso universal e igualitário a todos os níveis de assistência e prestar ações e serviços preventivos e curativos, individuais e coletivos (CAMPOS, 2003).

As medidas de prevenção se fazem necessárias porque, em algum momento, ao repetir o uso de uma ou mais substâncias, o "usuário" ao longo do tempo perde o controle sobre o comportamento de consumir drogas, tornando-se um "dependente", quando o desejo de consumir drogas torna-se compulsivo e muitas vezes irresistível, mesmo após um período sem fazer uso dela (O'BREIN \& McLEALLAN, 1996).

Basicamente, a prevenção pode ser das formas: primária (promoção à saúde), secundária (diminuição da incidência da doença real) e terciária (redução de comprometimento pela doença); porém, desde 1990, um segundo modelo tem sido usado para suplementar essa categoria de intervenção preventiva na saúde pública, podendo ser: universal (destinada à população geral), seletiva (com objetivo de atingir os grupos presumidamente de risco) e indicativo (para aqueles que já experimentaram drogas, por exemplo, mas talvez não se tornaram ainda dependentes) (OFFORD, 2000).

Logo, para que as conseqüências do uso nocivo de drogas causem o menor dano possível à população é necessário que se invista seriamente em todos os níveis de prevenção.

Um aspecto peculiar da prevenção é o fato de que a ação planejada deve ser aplicada a um grande número de pessoas, causar impacto, para, posteriormente, atingir cada indivíduo de forma efetiva. É um processo com resultado em longo prazo como citado por PETTA (2004), e deve começar pelo coletivo. Da mesma forma que o tratamento, depende da cultura, mídia, economia local, entre outros fatores. Apesar desta complexidade, os estudos têm mostrado que prevenir é melhor que tratar, principalmente quanto ao montante necessário para desenvolver essas ações.

O Ministério da Saúde, BRASIL (2003c) destaca que, a prevenção voltada para o uso abusivo e/ou dependência de álcool e outras drogas pode ser definida como um processo de planejamento, implantação e implementação de múltiplas estratégias voltadas para a redução dos fatores de vulnerabilidade e riscos específicos, e fortalecimento dos fatores de proteção. Implicando necessariamente em inserção comunitária das práticas propostas, como a colaboração de todos os segmentos sociais disponíveis, buscando atuar dentro de suas competências, para facilitar os processos que levem à redução da iniciação no consumo, do aumento deste em freqüência e intensidades, e das conseqüências do uso em padrões de menor comprometimento global.

Ainda, segundo o Ministério da Saúde, BRASIL (2003c), as ações preventivas devem facilitar a promoção global aos indivíduos, proporcionando ganhos de qualidade de vida das pessoas e oferecendo opções mais produtivas e alternativas ao uso de drogas enquanto fonte de prazer, e que detenham uma perspectiva evolutiva real ao futuro das pessoas.

A prevenção primária ao uso de álcool e outras drogas de acordo com PETTA (2004), tem no vértice social pelo menos quatro aspectos importantes: a família, a escola, o grupo e a comunidade. Somente através da articulação destes segmentos é que a prevenção primária ao uso de drogas pode ser tornar realmente efetiva.

Estudo de DISHION \& KAVANAGH (2000) aponta que com a interlocução entre escola e família podem ocorrer importantes contribuições para diminuir problemas comportamentais e uso de substâncias na perspectiva da saúde pública. Assim, ao considerar as particularidades de cada escola (BOTWIN, 2000), família, grupo e comunidade (CUIJIPERS \& SCIPPERS, 2002), na elaboração de programas de prevenção ao uso de drogas, os resultados se mostraram muito mais efetivos.

Para que a prevenção do primeiro uso de drogas se apresente de maneira realmente efetiva, GONÇALVES (2002) destaca que existe a necessidade de um trabalho em equipe multiprofissional em determinado contexto social; que, por um lado, deve romper a centralização do poder médico; e, por outro, desvie o enfoque curativo para questões de ordem social e para práticas educativas, enfatizando a promoção e a prevenção. No entanto, essa mudança no processo de trabalho não pode ser entendida como simplificação desse processo.

$\mathrm{Na}$ prevenção secundária, a atuação do profissional estaria direcionada às populações específicas numa abordagem mais objetiva. Um exemplo disso seria a oferta de uma assistência mais incisiva, ou seja, nos princípios da intervenção breve onde 0 profissional de saúde atuaria com a identificação do problema por meio de instrumentos 
de rastreamento do uso problemático do álcool (exemplo: AUDT, CAGE) e o aconselhamento para os riscos do uso das substâncias psicoativas, conhecimento das redes de referência e contrareferência, para os devidos encaminhamentos.

Para a prevenção terciária, os profissionais da atenção primária necessitariam de capacitação mais específica, que permitiria atuar no tratamento através de intervenções (motivações para mudanças de comportamentos, aconselhamentos e outros) que possibilitam o acompanhamento da evolução do cliente que já desenvolveram a dependência da droga e prevenindo complicações futuras, contribuindo também na reabilitação psicossocial e manutenção da abstinência, se for o caso.

Para tanto, como destaca PILLON (2003), para que estes níveis se estabeleçam na prática torna-se necessário uma ênfase no desenvolvimento de programas educacionais sobre álcool e outras drogas para profissionais de saúde atuarem tanto em níveis primários à saúde, quanto em níveis avançados e especializados nesta área.

É necessário que haja uma reorganização dos conteúdos teóricos e das práticas, incorporando criativamente os avanços técnico-científicos às bases teóricas e empíricas de cada contexto, social, cultural, particular, e epidemiológico. Para operacionalizar essas mudanças, como coloca GONÇALVES (2002), a formação de recursos humanos comprometidos integralmente com a interdisciplinaridade é fundamental, no sentido de uma troca de saberes e práticas que não aprisionam o processo de trabalho em estruturas rígidas; ao contrário, valoriza o potencial criativo e as competências de cada integrante da equipe.

Para que sejam trabalhadas a prevenção e a promoção à saúde na abordagem quanto ao uso de drogas, é preciso repensar a tradição organicista e tecnicista no tratamento dos problemas de saúde.

De acordo com LEVCOVITZ (1996), a concepção predominante na formação tradicional dos profissionais da área da saúde está sustentada pelo enfoque biologicista, centrado na doença e pouco vocacional ao social, conformando o modelo hegemônico no ensino e nas práticas de saúde, sendo que, se a formação dos profissionais de saúde não for modificada, o modelo de atenção também não o será, na realidade do dia-a-dia.

Profissionais habilitados tecnicamente em oferecer práticas transformadoras efetivas, com competência técnica, compromisso social e capacidade para trabalho em equipe multiprofissional estão ainda pouco disponíveis no mercado de trabalho, como cita GONÇALVES (2002). O modelo no qual os profissionais de saúde têm sido formados não está produzindo ações de acordo com necessidades da população, pouco tem articulado a educação e saúde, promoção, prevenção e reabilitação.

A educação formal sobre o uso de drogas e suas conseqüências apresenta limitações (PILLON, 2003). Para oferecer serviços com qualidade e compatíveis com a realidade, o profissional de saúde deveria estar preparado para atender às mudanças geradas nas necessidades de saúde.

Para concluir, um novo modelo de atenção à saúde tem sido proposto para se alcançar o desenvolvimento de um pensamento que responda pela complexidade que caracteriza o mundo atual, com seus desafios; entre eles, os problemas de saúde. E, para o alcance deste novo modelo são necessárias mudanças no sistema de formação dos profissionais de saúde (VILELLA \& MENDES, 2003)

\section{REFERÊNCIAS BIBLIOGRÁFICAS}

AALTO M; PEKURI P; SEPPÄ K. Primary health care professional activity in intervinng in patients alcohol drinssing: a patient perspective. Drug and Alcohol Dependence. v.66, p.39-43. 2002

BRASIL. Ministério da saúde. Programa Saúde da Família - PSF.Brasília: Ministério da Saúde. 2001.36p.

BRASIL. Ministério da Saúde.Departamento de Atenção Básica. Relatório de Gestão 2001. Brasília. 2003a. Disponível em: $\leq$ http:// www.saude.gov.br/departamentoeatençãobasica/relat oriodegestão2001>. [Acesso em 24 jun. 2003]

BRASIL. Ministério da Saúde. Informe Saúde. Brasília: Ministério da Saúde, v.7,n.197, 2003b.

BRASIL. Ministério da Saúde.Secretaria Executiva.Secretaria de Atenção à Saúde. Coordenação Nacional DST/AIDS. A Política do Ministério da Saúde para a Atenção Integral a Usuários de Álcool e Outras Drogas. Brasília: Ministério da Saúde.2003c.

BOTWIN, GJ. Preventing drug abuse in schools: social and competence enhancement approaches targenting individual level etiologic fators. Addictive Behaviors. v. 25, n. 6, p. 887-897, 2000.

CAMPOS, CEA. O desafio da integralidade segundo as perspectivas da vigilância da saúde e da saúde da família.Ciência \&Saúde Coletiva, v. 8, n. 2, p. 569584.2003.

CUIJPERS P; SCIPPERS GM. Integrating substance abuse treatament and preventionin the comunity. Addictive Behaviors. v. 27, p. 847-849. 2002.

DISHION, TJ; KAVANAGH, KA. Multilevel approach to family-centeared prevention in schools: process an outcome. Addictive Behaviors. v. 25, n. 6, p. 899911.2000.

GONÇALVES, AM. Cuidados diante do abuso e da dependência de drogas: um desafio da prática do programa saúde da família. 2002. 
209p.Tese.(Doutorado em Enfermagem Psiquiátrica)Escola de Enfermagem de Ribeirão Preto, Universidade de São Paulo, Ribeirão Preto, 2002.

SOUZA, HM. Incorporando uma prática. In: BRASIL, Seminário de Experiências Internacionais em Saúde da Família. 1999. Brasília. Relatório final. Brasília: Ministério da Saúde - Coordenação da Atenção Básica: 1999.

HENRY-EDWARDS, S; HUMENIUK, R; ALI, R; MONTEIRO, M; POZNYAK, V. Brief Intervention for Substance Use: A Manual for Use in Primary Care. (Draft Version 1.1 for Field Testing). Geneva, World Health Organization, 2003.

LEVCOVITZ E.; GARRIDO NG. Saúde da Família: a procura de um modelo anunciado. Cadernos de Saúde da Família. Brasília, v. 1, n. 1, p. 89-92, 1996.

O'BREIN \& McLEALLAN, A.T. Myths about the treatment of addiction.Lancet, v. 347, p. 237-40, 1996. OFFORD, DR. Selection of levels of prevention. Addictive Behaviors. v. 25, n. 6, p. 833-42, 2000.

PETTA, AC. Texto de aula. Mimeografado. 2004.

PILLON, SC. O uso de álcool a educação formal dos enfermeiros. 2003. 91p. Tese (Doutorado em Ciências) - Escola Paulista de Medicina, Universidade Federal de São Paulo, São Paulo.

TRAD, LAB.; BASTOS, ACS. O impacto sócio-cultural do Programa Saúde da Família (PSF): uma proposta de avaliação. Cadernos de Saúde Pública, Rio de Janeiro, v. 14, n. 2, p. 426-435, 1998.

SAMET JH; O'CONNOR, PG; STEIN, MD. Clínicas médicas da América do Norte: Abuso de álcool e de outras drogas. Rio de Janeiro: Inter-livros.1997.

VIANA, ALM; DAL POZ, MR. Reforma em saúde no Brasil: Programa de Saúde da Família; informe final. Estudos em Saúde Coletiva, Rio de Janeiro, n. 166, p. 3-35, 1998.

VILELLA, EM; MENDES, IJM. Interdisciplinaridade e saúde:estudo bibliográfico. Revista Latino-americana de Enfermagem, v. 11, n. 4, p. 531-537, 2003.

WORLD HEALTH ORGANISATION (WHO). Reducing Risks, promoting healthy life. The World Health Report. Geneva. 2002 